\title{
COLLOCATION METHOD BASED ON BARYCENTRIC INTERPOLATION ITERATION FOR ANALYSIS OF NONLINEAR MICROBEAMS
}

\author{
Meiling Zhuang, Changqing Miao \\ Key Laboratory of Concrete and Prestressed Concrete Structures of Ministry of Education, Southeast University, \\ Nanjing, China and \\ School of Civil Engineering, Southeast University, Nanjing, China \\ e-mail:18036558037@163.com; chqmiao@163.com (corresponging author)
}

ZHAOQING WANG

Institute of Mechanics, Shandong Jianzhu University, Jinan, China

\begin{abstract}
A powerful computational methodology, named the barycentric Lagrange interpolation iteration collocation method (BLIICM), for solving nonlinear bending problems of a doubly clamped microbeam under electrostatic loads is presented. The nonlinear governing equation of the microbeam is converted into a linear differential equation by assuming the initial function. The barycentric Lagrange interpolation collocation method (BLICM) is used to solve the linear differential equation. The direct linearization formulations and Newton linearization calculation formulations for the nonlinear differential equation have been given. The calculation method and formulation of the nonlinear integral term have been discussed in details. By applying a barycentric Lagrange interpolation differential matrix, a matrix-vector calculation formula of BLIICM has been established. Numerical results of calculation examples show that the advantages of the proposed methodology are efficient, simple and of high precision.
\end{abstract}

Keywords: nonlinear microbeams, barycentric Lagrange interpolation, collocation method, linearization method, iteration method

\section{Introduction}

Microbeams are used in microsensors and microactuators fabricated by MEMS technology (Brusa et al., 2004). The main mechanism of microsensor and microactuator can be simplified as a microbeam in micro electro-mechanical systems (MEMS). The geometric dimension of microbeams is normally in micron scale and microbeams mainly bear electric loads and axial forces. The relation between the force of electric potential acting on the microbeam and the deflection of the microbeam is nonlinear. For accurate analysis of microbeam mechanical properties, the elongation effect of the microbeam shall be considered. Considering external forces and geometric nonlinear factors of the microbeam, the governing equation of the microbeam bending established is a nonlinear differential equation. The analytical method used to solve the microbeam nonlinear differential equation is often extremely complicated and sometimes even impossible. With the progress of computer technology, the numerical method has become important for analysis of nonlinear bending of microbeams (Rezazadeh et al., 2009; Zand et al., 2009; Batra et al., 2008).

A high accuracy numerical method is needed to analyze microbeams, because the maximum deflection of the microbeams deformation is in micron to nanometer scales. Nayfeh, Mook, and Lobitz used a numerical-perturbation method to analyze forced vibration of a non-uniform beam (Nayfeh et al., 1974). Refwield (2015) analyzed vibration of nonlinear flat arch. Choi and Lovell 
(1997) converted the microbeam boundary value problem into the initial value problem and then used the shoot method to solve it (Choi, 1992). The differential quadrature method is a high precision collocation method widely used to solve Euler-Bernoulli microbeam bending and vibration problems (Shu and Du, 1997; Tomasiello, 1998; Karami and Malekzadeh, 2002). The differential quadrature method is a collocation method that approximates the unknown function based on Lagrange interpolation (Kuang and Chen, 2004; Najar et al., 2004; Sadeghian et al., 2007). Due to numerical instability of Lagrange interpolation, its calculation result tends to be instable with an increase of quantity of calculation nodes.

We can obtain a barycentric Lagrange interpolation by transforming the Lagrange interpolation into barycentric form. Rewriting the Lagrange interpolation formula to barycentric form can apparently improve numerical stability of the interpolation (Berrut and Trefethen, 2004). Wang et al. (2007) used the barycentric interpolation to approximate the unknown function, establishing differential matrices of the unknown function and each order of its derivative on calculation nodes, proposing a high accuracy grid-free barycentric interpolation collocation method to solve differential equation initial (boundary) value problems, and providing an algorithm program and a large amount of engineering calculation examples (Berrut et al., 2005; Floater and Hormann, 2007; Li and Wang, 2012; Wang et al., 2014a,b). Numerical calculation examples show that the barycentric interpolation collocation method has advantages of convenient use, high efficiency and high accuracy. It is a high accuracy meshless numerical calculation method applicable to numerical calculations for various differential equations.

Based on the barycentric interpolation collocation method and in combination with the linearization method for nonlinear differential equations, this article has established a barycentric Lagrange interpolation iteration collocation method (BLIICM) for solving nonlinear bending problems of doubly clamped microbeams under electrostatic loads, and presents numerical calculation examples to verify effectiveness and computational accuracy of this proposed method.

\section{Barycentric Lagrange interpolation and its differentiation matrices}

Given a function $v(x)$ defined on the interval $0=x_{1}<x_{2}<\cdots<x_{n}=l$ and function values on the nodes $v_{j}=v\left(x_{j}\right), j=1,2, \ldots, n$, the barycentric Lagrange interpolation of the function $v(x)$ is

$$
v(x)=\sum_{j=1}^{n} \frac{w_{j}}{x-x_{j}} v_{j} / \sum_{j=1}^{n} \frac{w_{j}}{x-x_{j}}
$$

where $w_{j}=1 / \prod_{j \neq k}\left(x_{j}-x_{k}\right), j=0,1, \ldots, n$ is the barycentric Lagrange interpolation weight. The barycentric Lagrange interpolation of the function $v(x)$ can be simplified as

$$
v(x)=\sum_{j=1}^{n} L_{j}(x) v_{j} \quad L_{j}(x)=\frac{w_{j}}{x-x_{j}} / \sum_{j=1}^{n} \frac{w_{j}}{x-x_{j}}
$$

And then, the $m$-th order derivative of the function $v(x)$ can be written as

$$
v^{(m)}(x)=\frac{d^{m} v(x)}{d x^{m}}=\sum_{j=1}^{n} L_{j}^{(m)}(x) v_{j}
$$

So the $m$-th order derivative of the function $v(x)$ on the nodes $x_{1}<x_{2}<\cdots<x_{n}$ can be written as

$$
v^{(m)}\left(x_{i}\right)=v_{i}^{(m)}=\frac{d^{m} v\left(x_{i}\right)}{d t^{m}}=\sum_{j=1}^{n} D_{i j}^{(m)} v_{j}
$$


Equation (2.4) can be written in the following matrix form (Wang et al., 2007)

$$
\mathbf{v}^{(m)}=\mathbf{D}^{(m)} \mathbf{v}
$$

where $\mathbf{v}^{(m)}=\left[v_{1}^{(m)}, v_{2}^{(m)}, \ldots, v_{n}^{(m)}\right]^{\mathrm{T}}$ and $\mathbf{v}=\left[v_{1}, v_{2}, \ldots, v_{n}\right]^{\mathrm{T}}$ represent the column vector of the $m$-th order derivative and the value of the function $v(x)$ on the nodes, respectively. Matrix $\mathbf{D}^{(m)}$ indicates the unknown function $m$-th order barycentric Lagrange interpolation differential matrix on the nodes $x_{1}, x_{2}, \ldots, x_{n}$, which is composed of the elements $D_{i j}^{(m)}=L_{j}^{(m)}\left(x_{i}\right)$.

\section{Calculation models of an MEMS microbeam}

As shown in Fig. 1, while a microbeam is loaded electrostatically, the transverse force per unit area is displacement dependent. So the function per unit force of the axial coordinate $x$ is expressed as

$$
p=p(v(x))=\frac{\varepsilon_{0} V^{2}}{2[g-v(x)]^{2}}
$$

where $V$ is the applied voltage, $g$ is the gap between the beams and the cover electrode (Fig. 1), and $\varepsilon_{0}=8.854 \cdot 10^{-12} \mathrm{~F} / \mathrm{m}$ is the permittivity of vacuum.

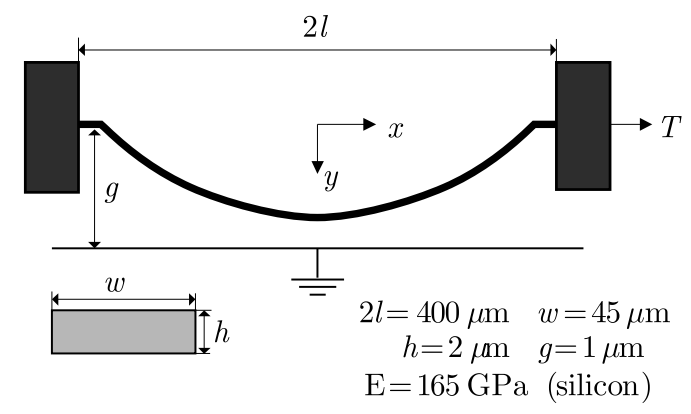

Fig. 1. Diagram of a doubly clamped microbeam

Stretching the induced tension $T$ and tension from the intrinsic strain $T_{i}$ can be included with the nonlinear loading for the most accurate representation (Nayfeh et al., 1974)

$$
E I \frac{d^{4} v}{d x^{4}}-\left(T+T_{i}\right) \frac{d^{2} v}{d x^{2}}=\frac{\varepsilon_{0} V^{2} w}{2[g-v(x)]^{2}}
$$

where $T_{i}$ is taken as $0.01 \%$ of the residual strain of the microbeam and $w$ is the width of the microbeam. According to the relation between the stretching tension and bending deflection of the microbeam, Eq. (3.2) can be written as

$$
E I \frac{d^{4} v(x)}{d x^{4}}-\left[\frac{E w h}{2 l} \int_{0}^{l}\left(\frac{d v(x)}{d x}\right)^{2} d x+T_{i}\right] \frac{d^{2} v(x)}{d x^{2}}=\frac{\varepsilon_{0} V^{2} w}{2[g-v(x)]^{2}}
$$

Equation (3.3) is solved by the numerical method proposed in this article with the boundary condition

$$
v^{\prime}(0)=v^{\prime}(2 l)=v(0)=v(2 l)=0
$$




\section{Linearization iteration collocation method for the nonlinear differential equation}

Using Eq. (2.2) to approximate the unknown function, nonlinear Eq. (3.4) is usually discreted to nonlinear algebraic equations and then the Newton-Raphson iteration is used to solve these nonlinear algebraic equations to obtain a numerical solution. The discrete form of the barycentric interpolation collocation method for Eq. (3.3) can be written as

$$
\begin{aligned}
E I & \sum_{j=1}^{n} L_{j}^{\prime \prime \prime \prime}\left(x_{i}\right) v_{j}-\left[\frac{E w h}{2 l} \int_{0}^{l}\left(\sum_{j=1}^{n} L_{j}^{\prime}(x) v_{j}\right)^{2} d x+T_{i}\right] \sum_{j=1}^{n} L_{j}^{\prime \prime}\left(x_{i}\right) v_{j} \\
& =\frac{\varepsilon_{0} V^{2} w}{2\left(g-\sum_{j=1}^{n} L_{j}(x) v_{j}\right)^{2}}
\end{aligned}
$$

It is very fussy to construct a Newton-Raphson iteration form of nonlinear Eq. (4.1). The linearization iteration collocation method is adopted to solve nonlinear Eq. (4.1). Under a mechanical load, for a given assumed initial function $v_{0}=v_{0}(x)$, under electric load and for the given assumed initial function of $v_{0}=v_{0}(x)$, Eq.(4.1) can be directly linearized to

$$
E I \frac{d^{4} v(x)}{d x^{4}}-\left[\frac{E w h}{2 l} \int_{0}^{l}\left(\frac{d v_{0}(x)}{d x}\right)^{2} d x+T_{i}\right] \frac{d^{2} v(x)}{d x^{2}}=\frac{\varepsilon_{0} V^{2} w}{2\left[g-v_{0}(x)\right]^{2}}
$$

Accordingly, the linearized iteration form can be directly constructed as follows

$$
E I \frac{d^{4} v_{k}(x)}{d x^{4}}-\left[\frac{E w h}{2 l} \int_{0}^{l}\left(\frac{d v_{k-1}(x)}{d x}\right)^{2} d x+T_{i}\right] \frac{d^{2} v_{k}(x)}{d x^{2}}=\frac{\varepsilon_{0} V^{2} w}{2\left[g-v_{k-1}(x)\right]^{2}} \quad k=1,2,3, \ldots
$$

Equation (4.3) is a linear differential equation. Using form (2.5) of the barycentric Lagrange interpolation and characteristic of interpolation basis functions, Eq. (4.3) can be written in form of a matrix

$$
\left[E I \mathbf{D}^{(4)}-\left(T_{k-1}+T_{i}\right) \mathbf{D}^{(2)}\right] \mathbf{v}_{k}=\mathbf{p}_{k-1} \quad k=1,2,3, \ldots
$$

where

$$
\mathbf{p}_{k-1}=\frac{\varepsilon_{0} V^{2} w}{2\left[g-\mathbf{v}_{k-1}\right]^{2}}
$$

is the external force vector of the $(k-1)$-th iteration.

The Newton iteration method is anthoer more available way. In the Newton iteration method, the approximating function is the line tangent to the nonlinear electric load

$$
p=p(v(x))=\frac{\varepsilon_{0} V^{2}}{2[g-v(x)]^{2}}
$$

at the initial point $\mathbf{v}_{0}$ (advised $\mathbf{v}_{0}=0$ ), where $v_{0}$ is the assumed initial point. Equation (4.2) in a Taylor series about $\mathbf{v}_{0}$ and discarding nonlinear terms yields

$$
p(v) \approx p\left(v_{0}\right)+p^{\prime}\left(v_{0}\right)\left(v-v_{0}\right)
$$


Substituting Eq. (4.5) into Eq. (4.2), we can obtain

$$
\begin{aligned}
& E I \sum_{j=1}^{n} L_{j}^{\prime \prime \prime \prime}\left(x_{i}\right) v_{j}-\left[\frac{E w h}{2 l} \int_{0}^{l}\left(\sum_{j=1}^{n} L_{j}^{\prime}(x) v_{j(0)}\right)^{2} d x+T_{i}\right] \sum_{j=1}^{n} L_{j}^{\prime \prime}\left(x_{i}\right) v_{j} \\
& \quad=p\left(v_{0}\right)+p^{\prime}\left(v_{0}\right)\left(v-v_{0}\right)
\end{aligned}
$$

Accordingly, the following iteration differential equation of the Newton linearization method can be obtained

$$
\begin{aligned}
& E I \sum_{j=1}^{n} L_{j}^{\prime \prime \prime \prime}\left(x_{i}\right) v_{j(k)}-\left[\frac{E w h}{2 l} \int_{0}^{l}\left(\sum_{j=1}^{n} L_{j}^{\prime}(x) v_{j(k-1)}\right)^{2} d x+T_{i}\right] \sum_{j=1}^{n} L_{j}^{\prime \prime}\left(x_{i}\right) v_{j(k)} \\
& \quad=p\left(v_{(k-1)}\right)+p^{\prime}\left(v_{(k-1)}\right)\left(v_{(k)}-v_{(k-1)}\right)
\end{aligned}
$$

Now, the iteration differential matrix form of Eq.(4.7) can be obtained as follows

$$
\left[E I \mathbf{D}^{(4)}-\left(T_{k-1}+T_{i}\right) \mathbf{D}^{(2)}\right] \mathbf{v}_{k}=\mathbf{p}_{k-1}+\mathbf{p}_{k-1}^{\prime}\left(\mathbf{v}_{k}-\mathbf{v}_{k-1}\right) \quad k=1,2,3, \ldots
$$

The nonlinear integral term in the microbeam governing Eq. (3.3) is related to stretching tension of the microbeam and its computational accuracy directly affects the error of the final computation result. We use Gauss integration for numerical integration. Barycentric interpolation of the unknown function is substituted in the integral term, and the square expansion of the integral term yields

$$
\begin{aligned}
T & =\frac{E w h}{2 l} \int_{0}^{l}\left(\frac{d v(x)}{d x}\right)^{2} d x=\frac{E w h}{2 l} \int_{0}^{l}\left(\sum_{j=1}^{n} L_{j}^{\prime}(x) v_{j}\right)^{2} d x \\
& =\frac{E w h}{2 l} \sum_{i=1}^{n} \sum_{j=1}^{n}\left(\int_{0}^{l} L_{i}^{\prime}(x) L_{j}^{\prime}(x) d x\right) v_{i} v_{j}
\end{aligned}
$$

Introducing notation $A_{i j}=\int_{a}^{b}\left[L_{i}^{\prime}(x) L_{j}^{\prime}(x)\right] d x$, we can obtain

$$
\int_{a}^{b}\left(\frac{d v}{d x}\right)^{2} d x=\sum_{i=1}^{N} \sum_{j=1}^{N} A_{i j} v_{i} v_{j}=\mathbf{v}^{\mathrm{T}} \mathbf{A} \mathbf{v}
$$

where $\mathbf{A}$ is an $n \times n$ matrix comprising elements $A_{i j}$ and referred to as the barycentric interpolation integral matrix. $A_{i j}$ is a definite integral of arycentric interpolation primary function derivative interactive product, which is constant only if related to the interpolation node and irrelevant of the type of problem to be solved. According to formula (4.10), we can obtain stretching tension of the microbeam after $(k-1)$-th iteration

$$
T_{k-1}=\frac{E w h}{2 l} \int_{0}^{l}\left(\frac{d v_{k-1}(x)}{d x}\right)^{2} d x=\frac{E w h}{2 l} \mathbf{v}_{k-1}^{\mathrm{T}} \mathbf{A v}_{k-1}
$$

The substitution method (Wang et al., 2007) is used to apply boundary conditions of the MEMS microbeam. Starting from the assumed initial value $\mathbf{v}_{0}\left(\mathbf{v}_{0}=\mathbf{0}\right)$ the iteration method is used to solve direct linearization Eq. (4.4) or Newton linearization Eq. (4.8), to obtain a corrected solution $\mathbf{v}_{1}$ of the unknown function $v(x)$ on nodes. Then, Eq. (4.11) can be used to obtain the corrected solution $T_{1}$. For a given control accuracy $\varepsilon=10^{-10}$, if $\left\|\mathbf{v}_{k}-\mathbf{v}_{k-1}\right\|_{\infty}<\varepsilon$, the corrected solution $\mathbf{v}_{k}$ is the numerical solution of the MEMS microbeam; otherwise the iteration is kept until the computational control accuracy $\varepsilon$ is met and then the numerical solution is obtained. 


\section{Numerical results}

The calculation program is compiled by MATLAB. By making use of the barycentric Lagrange interpolation differential matrix, the matrix-vector calculation formula of BLIICM has been established. Relying on the powerful matrix operation capability of MATLAB, the calculation program can be easily and quickly compiled. Microbeam geometric and physical parameters are shown in Fig. 1. For calculation, the computational domain is $[0,400]$ and the type of discrete nodes is the Chebyshev node $x_{i}=200+200 \cos (i \pi / n), i=0,1,2, \ldots, n$. In the collocation method, 21 nodes are selected. The control precision of iteration methods is $10^{-10}$ and the Gauss integral with 6 points is applied.

In this Section, there is no analytical solution to Eq. (3.2). So, we quote the maximum residual error (err) to illustrate the solution accuracy of BLIICM. Here, the maximum residual error is

$$
\operatorname{err}=\max _{k=1,2, \ldots, n}\left|E I v^{(4)}\left(x_{k}\right)-\left(T+T_{i}\right) v^{(2)}\left(x_{k}\right)-p\left(v\left(x_{k}\right)\right)\right|
$$

Based on the numerical results of Fig. 2, Table 1 and Table 2 comprehensively, the following observations can be concluded:

- in terms of the convergence rate, the Newton linearization iteration is apparently faster than the direct linearization iteration,

- in terms of differential equation residual, the Newton linearization iteration method has a higher computational accuracy than the direct linearization iteration method,

- computation results of BLIICM are highly consistent with the results of reference.
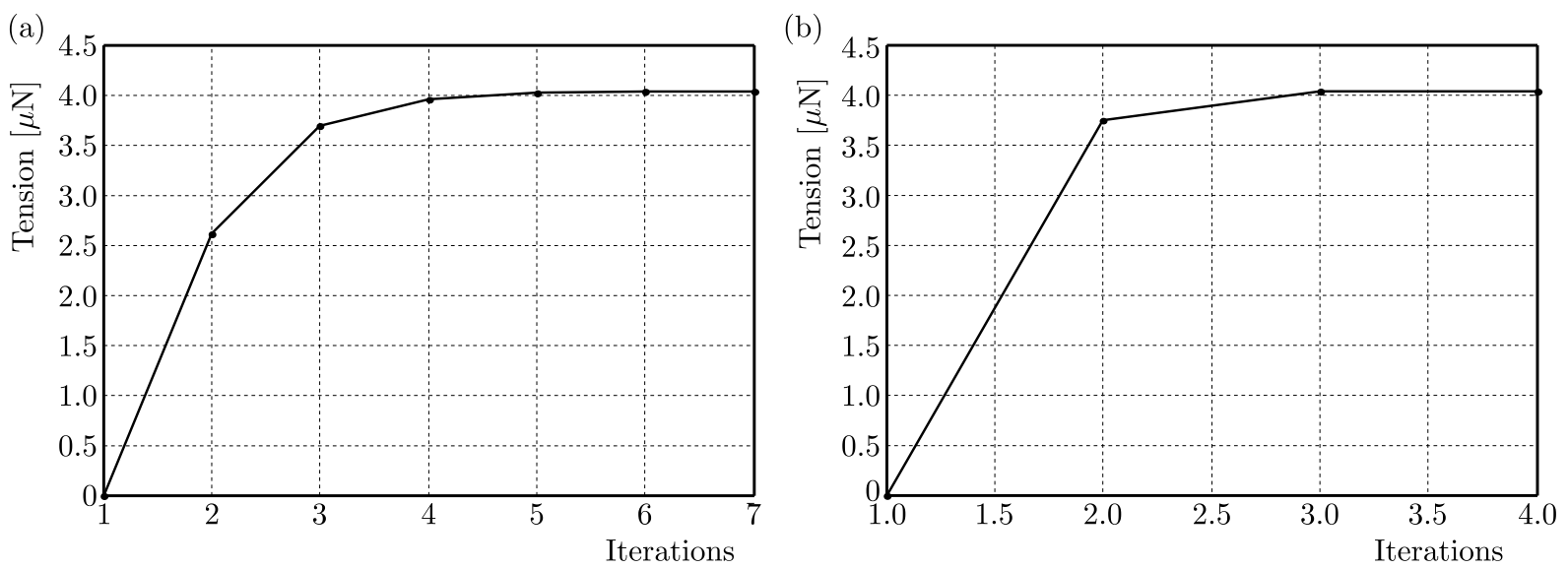

Fig. 2. The iterative process of the direct (a) and Newton (b) linearization method at $V_{2}=40$

Figure 3 shows comparative results of the maximum deflection of the microbeam for three tensional cases with various voltages. It can be seen that the existance of induced tension strengthens the deformation resistance of the microbeam. It is also known that deflection of the microbeam is the smallest while considering the induced tension and the residual tensile strain of $0.01 \%$, and that the deflection of the microbeam is the largest when the axial effects are absent.

Figure 4 shows the relations of the induced tensile force and residual strain of the microbeam with various voltages. It can be seen that the residual stain has a huge effect on the induced tensile force, and this effect is more remarkable with an increase of the electric load. The mechanical properties of the microbeam caused by the residual stain are known that an extremely high dimensional precision applies to the process and installation of the microbeam, and it needs to have a great improvement to the process level in fact. 
Table 1. MEMS microbeam calculation results without considering $0.01 \%$ residual strain

\begin{tabular}{|c|c|c|c|c|c|c|c|c|}
\hline \multirow{2}{*}{$\begin{array}{c}\text { Vol- } \\
\text { tage } \\
{\left[\mathrm{V}^{2}\right]}\end{array}$} & \begin{tabular}{c}
$|c|$ \\
\cline { 2 - 9 } \\
deflection \\
{$[\mu \mathrm{m}]$}
\end{tabular} & $\begin{array}{c}\text { Axial } \\
\text { force } \\
{[\mu \mathrm{N}]}\end{array}$ & $\begin{array}{c}\text { Itera- } \\
\text { tions }\end{array}$ & $\begin{array}{c}\text { Residual } \\
\text { error }\end{array}$ & $\begin{array}{c}\text { Midspan } \\
\text { deflection } \\
{[\mu \mathrm{m}]}\end{array}$ & $\begin{array}{c}\text { Axial } \\
\text { force } \\
{[\mu \mathrm{N}]}\end{array}$ & $\begin{array}{c}\text { Itera- } \\
\text { tions }\end{array}$ & $\begin{array}{c}\text { Residual } \\
\text { error }\end{array}$ \\
\hline \hline 20 & 0.0589 & 0.7843 & 4 & $4.0536 \cdot 10^{-7}$ & 0.0589 & 0.7845 & 3 & $2.5976 \cdot 10^{-15}$ \\
\hline 30 & 0.0935 & 1.9791 & 5 & $6.4801 \cdot 10^{-7}$ & 0.0936 & 1.9812 & 3 & $1.2745 \cdot 10^{-14}$ \\
\hline 40 & 0.1335 & 4.0329 & 7 & $1.3098 \cdot 10^{-6}$ & 0.1335 & 4.0359 & 4 & $5.0783 \cdot 10^{-12}$ \\
\hline 50 & 0.1818 & 7.4771 & 8 & $1.3105 \cdot 10^{-6}$ & 0.1819 & 7.4891 & 4 & $1.3164 \cdot 10^{-14}$ \\
\hline 60 & 0.2463 & 13.7156 & 10 & $5.3423 \cdot 10^{-6}$ & 0.2463 & 13.7265 & 4 & $5.2615 \cdot 10^{-12}$ \\
\hline
\end{tabular}

Table 2. BLIICM and Shooting Method numerical results of the MEMS microbeam without considering $0.01 \%$ residual strain

\begin{tabular}{|c|c|c|c|c|c|c|}
\hline \multirow{2}{*}{$\begin{array}{l}\text { Vol- } \\
\text { tage }\end{array}$} & \multicolumn{2}{|c|}{ Direct iteration method } & \multicolumn{2}{|c|}{ Newton iteration method } & \multicolumn{2}{|c|}{$\begin{array}{c}\text { Shooting method, } \\
\text { Zand } \text { et al. }(2009)\end{array}$} \\
\cline { 2 - 7 }$\left[\mathrm{V}^{2}\right]$ & $\begin{array}{c}\text { Midspan } \\
\text { deflection } \\
{[\mu \mathrm{m}]}\end{array}$ & $\begin{array}{c}\text { Axial } \\
\text { force } \\
{[\mu \mathrm{N}]}\end{array}$ & $\begin{array}{c}\text { Midspan } \\
\text { deflection } \\
{[\mu \mathrm{m}]}\end{array}$ & $\begin{array}{c}\text { Axial } \\
\text { force } \\
{[\mu \mathrm{N}]}\end{array}$ & $\begin{array}{c}\text { Midspan } \\
\text { deflection } \\
{[\mu \mathrm{m}]}\end{array}$ & $\begin{array}{c}\text { Axial } \\
\text { force } \\
{[\mu \mathrm{N}]}\end{array}$ \\
\hline \hline 1.0 & $0.2694 \cdot 10^{-2}$ & $0.1629 \cdot 10^{-2}$ & $0.2694 \cdot 10^{-2}$ & $0.1643 \cdot 10^{-2}$ & $0.2694 \cdot 10^{-2}$ & $0.1642 \cdot 10^{-2}$ \\
\hline 2.0 & $0.1091 \cdot 10^{-1}$ & $0.2686 \cdot 10^{-1}$ & $0.1091 \cdot 10^{-1}$ & $0.2678 \cdot 10^{-1}$ & $0.1089 \cdot 10^{-1}$ & $0.2677 \cdot 10^{-1}$ \\
\hline 3.0 & $0.2512 \cdot 10^{-1}$ & $0.1425 \cdot 10^{0}$ & $0.2512 \cdot 10^{-1}$ & $0.1427 \cdot 10^{0}$ & $0.2512 \cdot 10^{-1}$ & $0.1427 \cdot 10^{0}$ \\
\hline 4.0 & $0.4617 \cdot 10^{-1}$ & $0.4821 \cdot 10^{0}$ & $0.4617 \cdot 10^{-1}$ & $0.4822 \cdot 10^{0}$ & $0.4617 \cdot 10^{-1}$ & $0.4822 \cdot 10^{0}$ \\
\hline 5.0 & $0.7570 \cdot 10^{-1}$ & $0.1296 \cdot 10^{1}$ & $0.7570 \cdot 10^{-1}$ & $0.1297 \cdot 10^{1}$ & $0.7570 \cdot 10^{-1}$ & $0.1297 \cdot 10^{1}$ \\
\hline 6.0 & $0.1168 \cdot 10^{-1}$ & $0.3083 \cdot 10^{1}$ & $0.1168 \cdot 10^{-1}$ & $0.3085 \cdot 10^{1}$ & $0.1168 \cdot 10^{-1}$ & $0.3085 \cdot 10^{1}$ \\
\hline 7.0 & $0.1765 \cdot 10^{0}$ & $0.7039 \cdot 10^{1}$ & $0.1765 \cdot 10^{0}$ & $0.7046 \cdot 10^{1}$ & $0.1765 \cdot 10^{0}$ & $0.7045 \cdot 10^{1}$ \\
\hline 8.0 & $0.2821 \cdot 10^{0}$ & $0.1798 \cdot 10^{2}$ & $0.2822 \cdot 10^{0}$ & $0.1800 \cdot 10^{2}$ & $0.2822 \cdot 10^{0}$ & $0.1800 \cdot 10^{2}$ \\
\hline
\end{tabular}

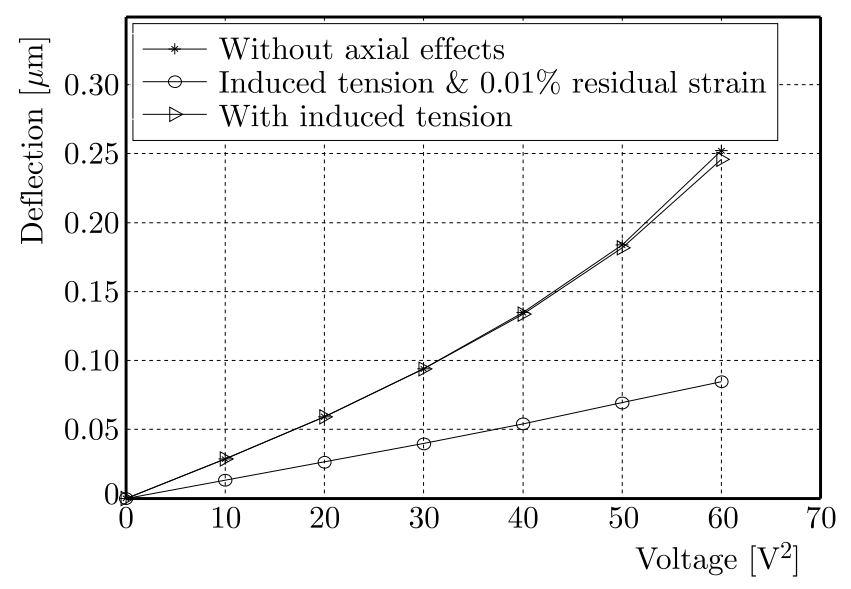

Fig. 3. Maximum deflections for electrical loading and three tensional cases with various voltages

\section{Conclusions}

Doubly clamped microbeams under electrostatic loads have been analysed by using the collocation method based on the barycentric Lagrange interpolation iteration. Compared with other numerical methods of solving the differential equation, the collocation method in this article has merits of simple calculation formulations, convenient program and a high computation preci- 


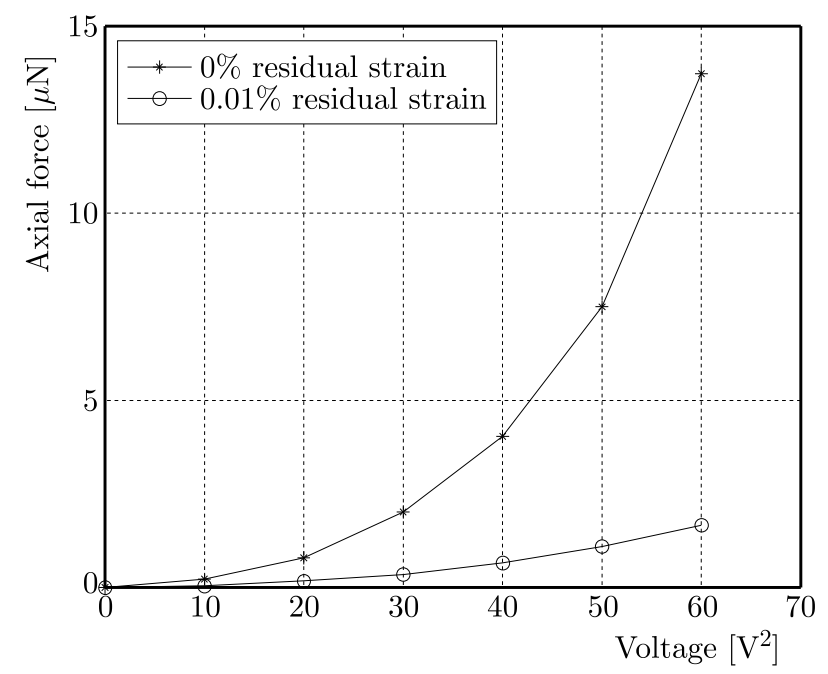

Fig. 4. Induced axial force for electrical loading with and without the residual strain with various voltages

sion. The BLIICM adopting matrix-vector calculation formula has obvious advantages in solving nonlinear problems as it can analyze such problems quickly and accurately.

For the existing stretching effects, the induced tension has a considerable influence in reducing displacements and stresses because the stiffness increases with the increasing transverse load. In a especial case of electrical loading, the residual strain has a strong influence on the induced tensile force. Therefore, analysis of the microbeam geometric nonlinear factor and the inertial nonlinear factor of MEMS microbeam mechanical properties is very important. In particular, the microbeam geometric nonlinear factor and the inertial nonlinear factor are decisive for design and use in MEMS, and sufficient attention should be given to them before implementation.

\section{Acknowledgements}

This research has been supported by the National Natural Science Foundation of China under grant No. 51778135 and Aeronautical Science Foundation of China under grant No. 20130969010. This research also has been supported by the Fundamental Research Funds for the Central Universities and Postgraduate Research and Practice Innovation Program of Jiangsu Province under grant No. KYCX18_0113.

\section{References}

1. Batra R.C., Porfir M., Spinello D., 2008, Vibrations of narrow microbeams predeformed by an electric field, Journal of Sound and Vibration, 309, 3-5, 600-612

2. Berrut J.P., Baltensperger R., Mittelmann H.D., 2005, Recent developments in barycentric rational interpolation, [In:] Trends and Applications in Constructive Approximation, D.H. Mache, J. Szabados, M.G. de Bruin (Edit), 27-51

3. Berrut J.P., Trefethen L.N., 2004, Barycentric Lagrange interpolation, SIAM Review, 46, 3, $501-517$

4. Brusa E., Bona F.D., Gugliotta A., 2004, Modeling and prediction of the dynamic behaviour of microbeams under electrostatic load, Analog Integrated Circuits and Signal Processing, 40, 2, 155-164

5. CHoi B., 1992, Diaphragm Stretching and Contact Problems for Microelectromechanical Pressure Transducers, Madison: University of Wisconsin

6. Choi B., Lovell E.G., 1997, Improved analysis of microbeams under mechanical and electrostatic loads, Journal of Micromechanics and Microengineering, 7, 1, 24-29 
7. Floater M.S., Hormann K., 2007, Barycentric rational interpolation with no poles and high rates of approximation, Numerische Mathematik, 107, 2, 315-331

8. Karami G., Malekzadeh P., 2002, A new differential quadrature methodology for beam analysis and the associated differential quadrature element method, Computer Methods in Applied Mechanics and Engineering, 191, 32, 3509-3526

9. KuAng J.H., Chen C.J., 2004, Dynamic characteristics of shaped micro-actuators solved using the differential quadrature method, Journal of Micromechanics and Microengineering, 14, 4, 647-655

10. Li S.C., Wang Z.Q., 2012, Algorithm, Program and Engineering Application on High Precision and Meshless Barycentric Interpolation Collocation Method, Beijing: Science Press

11. Najar F., Choura S., Elborgi S., Abdelrahman E.M., Nayfeh A.H., 2004, Modeling and design of variable-geometry electrostatic microactuators, Journal of Micromechanics and Microengineering, 15, 3, 419-429

12. Nayfeh A.H., Mook D.T., Lobitz D.W., 1974, Numerical-perturbation method for the nonlinear analysis of structural vibrations, AIAA Journal, 12, 9, 1222-1228

13. Refwield L.W., 2015, Nonlinear flexural oscillations of shallow arches, AIAA Journal, 12, 1, 91-93

14. Rezazadeh G., Tahmasebi A., Ziaei-Rad S., 2009, Nonlinear electrostatic behavior for two elastic parallel fixed-fixed and cantilever microbeams, Mechatronics, 19, 6, 840-846

15. Sadeghian H., Rezazadeh G., Osterberg P.M., 2007, Application of the generalized differential quadrature method to the study of pull-in phenomena of MEMS switches, Journal of Microelectromechanical Systems, 16, 6, 1334-1340

16. Shu C., Du H., 1997, Implementation of clamped and simply supported boundary conditions in the GDQ free vibration analysis of beams and plates, International Journal of Solids and Structures, 34, 7, 819-835

17. Tomasiello S., 1998, Differential quadrature method application to initial-boundary-value problems, Journal of Sound and Vibration, 218, 4, 573-585

18. WAng Z.Q., Jiang J., TAng B.T., Zheng W., 2014, Numerical solution of bending problem for elliptical plate using differentiation matrix method based on barycentric Lagrange interpolation, Applied Mechanics and Materials, 638-640, 1720-1724

19. WANG Z.Q., Li S.P., TANG B.T., 2007., Formulations, algorithms and applications on barycentric interpolation in 1D, Journal of Shandong Jianzhu University, 22, 4, 448-453

20. Wang Z.Q., Li S.C., Ping Y., JiAng J., MA T.F., 2014, A highly accurate regular domain collocation method for solving potential problems in the irregular doubly connected domains, $M a$ thematical Problems in Engineering, 2014, 4, 1-9

21. Zand M.M., Ahmadian M.T., Rashidian B., 2009, Semi-analytic solutions to nonlinear vibrations of microbeams under suddenly applied voltages, Journal of Sound and Vibration, 325, 1, $382-396$ 